lymphoid follicles [7]. Furthermore, the significantly higher pulmonary Treg cell numbers in air- and CS-exposed CCR7 ${ }^{-/-}$ mice correlated with a stronger abundance of lymphoid follicles, compared with WT animals (fig. $1 \mathrm{~b}$ and [7]). In contrast, baseline and chronic CS-induced Treg cell accumulation were severely compromised in lymph nodes of CCR $7^{-1}$ mice compared to WT controls (fig. 1c).

Our study provides evidence that CS exposure induces increased Treg cell numbers, first in the lung and secondly in the lymph node compartment. Whereas CCR7 is crucial for the homing of Treg cells to the lymph nodes, it is ultimately not required for the chronic CS-induced accumulation of these cells in the lung. However, we can not differentiate whether the observed CS-induced Treg increase results from the recruitment of natural Treg cells as opposed to the local induction of Treg cells from precursors. The methylation status of the FOXP3 promoter is generally considered to discriminate between natural and induced Treg cells.

Further translational research is needed to elucidate the functional role of lymphoid follicles and Treg cells in the pathogenesis of COPD, for instance by determining the contribution of natural committed Treg cells versus induced Treg cells in pulmonary lymphoid follicles and lymph nodes.

\section{T. Demoor, K.R. Bracke, G.F. Joos and G.G. Brusselle}

Dept of Respiratory Medicine, Laboratory for Translational Research in Obstructive Pulmonary Diseases, Ghent University Hospital, Ghent, Belgium.
Correspondence: G.G. Brusselle, Dept of Respiratory Medicine, Ghent University Hospital 7K12E, De Pintelaan 185, 9000 Ghent, Belgium. E-mail: guy.brusselle@ugent.be

Statement of Interest: None declared.

\section{REFERENCES}

1 Hogg JC, Chu F, Utokaparch S, et al. The nature of small-airway obstruction in chronic obstructive pulmonary disease. $N$ Engl J Med 2004; 350: 2645-2653.

2 van der Strate BW, Postma DS, Brandsma CA, et al. Cigarette smokeinduced emphysema: A role for the B cell? Am J Respir Crit Care Med 2006; 173: 751-758.

3 Brusselle GG, Demoor T, Bracke KR, et al. Lymphoid follicles in (very) severe COPD: beneficial or harmful? Eur Respir J 2009; 34: 219-230.

4 Plumb J, Smyth LJ, Adams HR, et al. Increased T-regulatory cells within lymphocyte follicles in moderate COPD. Eur Respir J 2009; 34: 89-94.

5 D'hulst AI, Vermaelen KY, Brusselle GG, et al. Time course of cigarette smoke-induced pulmonary inflammation in mice. Eur Respir J 2005; 26: 204-213.

6 Demoor T, Bracke KR, Maes T, et al. Role of lymphotoxin- $\alpha$ in cigarette smoke-induced inflammation and lymphoid neogenesis Eur Respir J 2009; 34: 405-416.

7 Demoor T, Bracke KR, Vermaelen KY, et al. CCR7 modulates pulmonary and lymph node inflammatory responses in cigarette smoke-exposed mice. J Immunol 2009; 183: 8186-8194.

\title{
Diagnostic accuracy of digital chest radiography for pulmonary tuberculosis in a UK urban population
}

\section{To the Editors:}

Population screening for tuberculosis was discontinued in most Western countries, largely due to the decreasing prevalence of the disease. The use of chest radiograph examination for population screening has, therefore, appropriately declined and is now limited to the screening of high-risk groups, such as immigrants [1], prisoners or homeless persons in certain countries [2] and for disease prevalence surveys [3]. Chest radiography, however, remains a key tool for the clinical diagnosis of pulmonary tuberculosis. Previous studies suggest that chest radiograph abnormalities in tuberculosis are not specific and levels of intra- and inter-reader agreement are very variable [4-6]. Modern digital tuberculosis screening has been noted to have high levels of sensitivity where chest radiograph examination is standardised, quality assured and a simple coding system is used [7-9]. Levels of reader agreement are also reported to be higher among experienced readers [10].

In many low incidence countries, tuberculosis is concentrating in specific urban populations, such as homeless persons and drug users, with high prevalence rates reported [2, 11]. In the $\mathrm{UK}$, this may be contributing to the general rise in tuberculosis [12]. Using a sample of digital chest radiographs from a hard to reach population at high risk of tuberculosis in London, we assessed some of the factors associated with greater diagnostic accuracy and determined levels of agreement between readers.

The study was carried out in London (UK), Amsterdam and Rotterdam (the Netherlands) between July and October 2008. Of 20 physicians invited, 13 participated: three radiologists, six respiratory physicians and one infectious disease physician from London and three public health tuberculosis physicians from Amsterdam and Rotterdam. 56 randomly selected chest radiographs were chosen: 18 confirmed cases of tuberculosis, 19 other abnormalities and 19 normal films from a screening programme which targeted homeless persons, problem drug users and prisoners. All cases had culture confirmed disease and eight were sputum smear positive. Study participants reviewed images using a DICOM (digital imaging and communications in medicine) viewer in a darkened room 
during a single viewing session, independently from each other. Participants were allowed $2 \mathrm{~min}$ to read each film and $1 \mathrm{~min}$ to complete a standard pro forma [13]. Blind to the true diagnosis, readers categorised chest radiograph findings on each film as normal, abnormal or other. If abnormal, information was requested on whether the findings were suggestive of active tuberculosis or inactive tuberculosis [13]. We also collected information from participants on the number of years of experience, speciality, annual number of tuberculosis cases notified and chest radioghraphs read in the previous year.

As culture confirmation was available on all chest radiographs included as having tuberculosis, we used this as the gold standard to calculate diagnostic odds ratios overall and by speciality. To assess the effect of the characteristics of readers, the logarithm of the diagnostic odds ratio for each participant was regressed against reader characteristics to determine its effect. The estimates were then anti-logged to give fold effects of the reader characteristics, including speciality type, number of years of experience, number of notifications and country of practice, on the diagnostic odds ratios.

To investigate the effect of patient characteristics on diagnostic accuracy with culture confirmation as a gold standard, we undertook sample level analysis where each film is a unit of observation. The number of readers who correctly diagnosed each film was determined and compared by the patient's age group, sex and sputum smear positive status using the Kruskal-Wallis test.

We used the kappa statistic (two outcomes, multiple readers) with $95 \%$ confidence intervals to assess inter-reader agreement among the participants.

The 13 readers assessed a median (range) of 52 (10-450) notified cases per year and had a median speciality experience of 15 (8-29) yrs. The median (interquartile range) age of the population from whom chest radiographs were sampled was 41 (29-49) yrs and 82\% (46) were male.

The mean diagnostic odds ratio for all participants for correctly identifying a patient with culture confirmed tuberculosis was 17.1 (95\% CI 12.3-23.6). Based on an overall sensitivity of $77 \%$ and specificity of 95\%, 497 individuals without tuberculosis will be referred compared to 61 correctly diagnosed cases of tuberculosis among 10,024 homeless persons in London. The mean diagnostic accuracy was high for physicians (16.8, 95\% CI 10.7-26.2) and radiologists (17.7, 95\% CI 8.0-39.3). Figure 1 shows the receiver operating characteristic curve by speciality. However, the diagnostic accuracy did not differ significantly by speciality or country $(\mathrm{p}>0.05)$. More years in a speciality was associated with a $1.23(95 \%$ CI $1.02-1.48)$ fold increase in diagnostic odds ratio per 10 yrs of identifying cases of tuberculosis $(p=0.033)$ (table 1$)$. Similarly, participants with greater number of notifications per year were more likely to correctly identify tuberculosis cases (OR 3.07, 95\% CI 1.08-8.69 per 1000 notifications, $\mathrm{p}=0.037$ ).

Participants were more likely to get the correct diagnosis when the individuals screened were younger (median number of correct diagnoses 13 versus 12; $\mathrm{p}=0.024$ ) or had sputum smear positive disease (median number of correct diagnoses

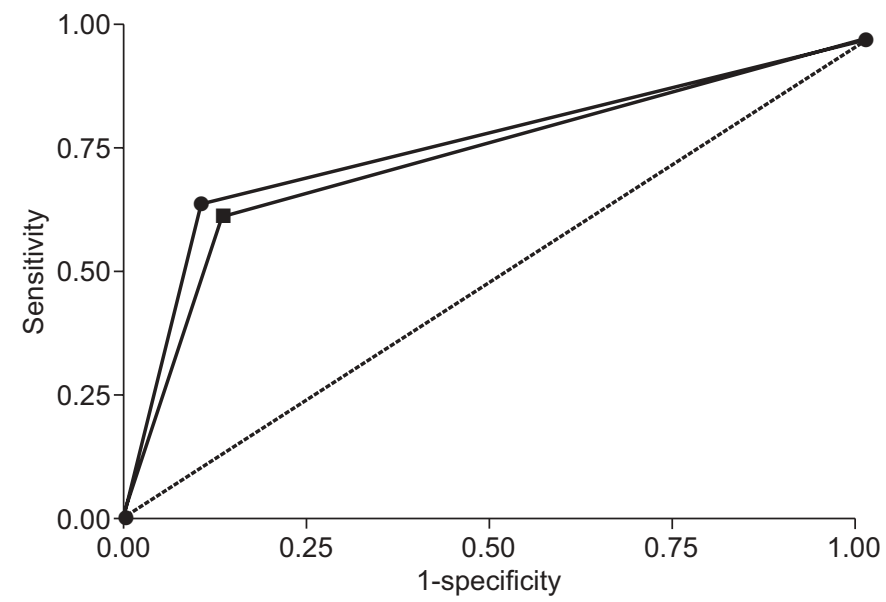

FIGURE 1. Receiver operating characteristic curve comparing diagnostic accuracy for tuberculosis by speciality. $\mathbf{\square}$ : radiologists; $\bullet$ : physicians.

13 versus six; $\mathrm{p}=0.028)$. There was no difference by sex $(\mathrm{p}=0.677)$.

The overall level of agreement for diagnosing active tuberculosis was good, with a kappa value of 0.61 (95\% CI $0.48-0.73$ ) and slightly higher between physicians (0.64) compared to radiologists (0.54). In contrast, radiologists were significantly more likely to agree on a normal chest radiograph with a very good level of agreement of 0.84 (95\% CI 0.68-0.95) when compared to physicians (kappa $0.46,95 \%$ CI $0.32-0.55$ ). The overall level of agreement was good for identifying the presence of infiltrates, consolidation or pleural effusion and moderate for the identification of cavities (table 2). Radiologists were very good at identifying cavities and pleural effusions, with kappa values found to be 0.70 and 1 respectively.

In contrast to previous reports, the number of chest radiographs examined per year leading to an improved diagnostic accuracy was lower than the 20,000 films previously reported [14]. Physicians were particularly good at diagnosing tuberculosis, while radiologists were very good at detecting a normal chest radiograph.

\begin{tabular}{|c|c|c|}
\hline Characteristic of reader & $\begin{array}{l}\text { Fold effect on diagnostic } \\
\text { OR }(95 \% \mathrm{Cl})\end{array}$ & p-value \\
\hline \multicolumn{3}{|l|}{ Speciality type } \\
\hline Radiologists & 1.0 (baseline) & \\
\hline Physicians & $0.96(0.71-1.35)$ & 0.871 \\
\hline \multicolumn{3}{|l|}{ Years in speciality } \\
\hline Per 10 yrs in speciality & $1.23(1.02-1.48)$ & 0.033 \\
\hline \multicolumn{3}{|l|}{ Number of notifications } \\
\hline Per 1000 notifications & $3.07(1.08-8.69)$ & 0.037 \\
\hline \multicolumn{3}{|l|}{ Country } \\
\hline UK & 1.0 (baseline) & \\
\hline The Netherlands & $1.10(0.78-1.56)$ & 0.552 \\
\hline
\end{tabular}




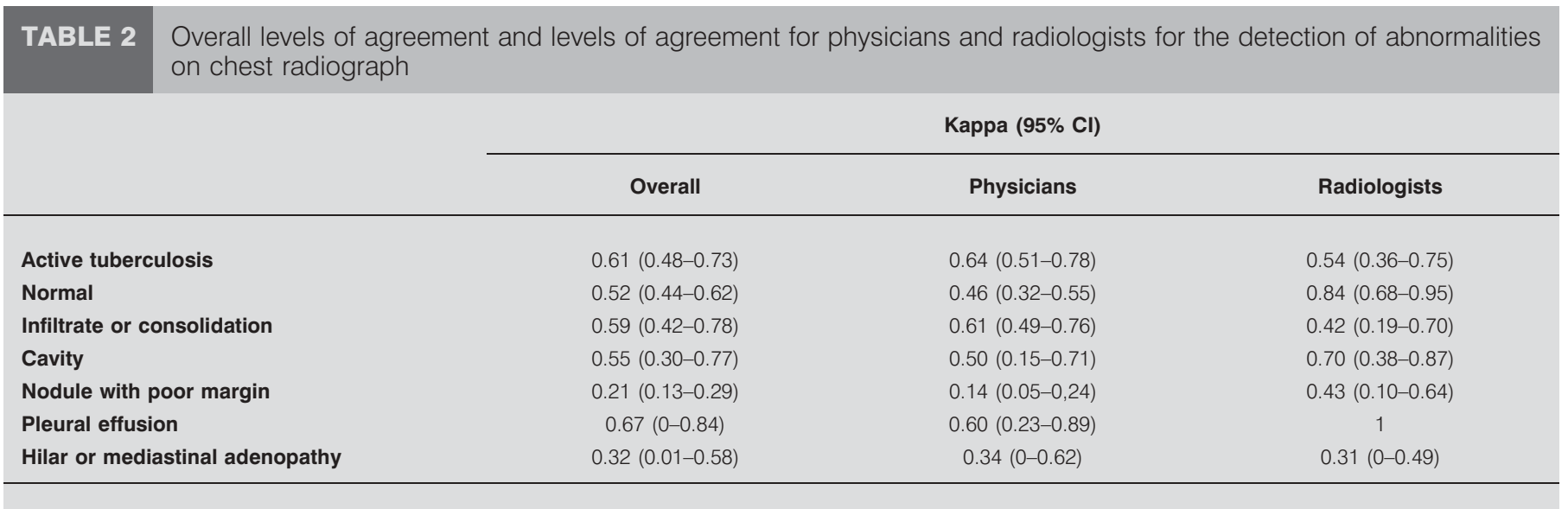

Similarly high levels of inter-reader agreement have been reported from Switzerland among experienced readers [10] and from South Africa, where a standardised method for reading and reporting was used [8]. In contrast, a Russian study [4] reported fair to moderate agreement between 101 clinicians using a non-diagnostic standard viewing platform, although levels of agreement were still higher among more experienced physicians.

Chest radiography will only be useful as a tool for tuberculosis screening if it has high diagnostic accuracy when applied to a high prevalence population. In contrast to older studies using conventional radiography for mass population screening [15], we found the diagnostic accuracy of digital radiography in this population to be high, which is consistent with more recent reports [7]. Previous estimates, for example homeless persons (prevalence 788 per 100,000), problem drug users (354 per $100,000)$ and prisoners (208 per 100,000), suggest these groups may be suitable for screening [2]. Using diagnostic accuracy data from this study and published prevalence of tuberculosis among homeless persons [2], we estimate the positive predictive value of chest radiograph screening to be $11 \%$ in this population.

Not surprisingly, previous studies suggest that chest radiograph screening of immigrants from high tuberculosis burden countries is not clinically or cost effective [16]. The prevalence of tuberculosis in immigrants depends on their country of origin. It is also difficult to distinguish some cases of active from inactive tuberculosis based on a single radiological film. In contrast, the use of chest radiograph screening among groups with very high incidence of tuberculosis in metropolitan areas aids the identification of pulmonary disease, therefore contributing to halting transmission. A recent Dutch study using DNA fingerprinting demonstrated that mobile digital radiography contributed to a reduction in tuberculosis transmission among groups such as homeless persons and drug users [11]. Furthermore, alternative interventions, such as preventive treatment of latent tuberculosis infection, can be challenging in groups such as prisoners and homeless persons [17].

Only 13 readers participated and 56 films were used, possibly limiting our ability to detect significant variation. The number of films is, however, comparable to the Russian study [4] and more readers participated than in other similar studies $[8,10]$. Our study did not include an intra-reader agreement component. Reports from previous studies suggest moderate [4] to high levels of intra-reader agreement [10].

An often cited concern regarding the feasibility of screening is the excess activity generated from evaluating a large number of individuals suspected of, but without, tuberculosis [4]. Based on the diagnostic accuracy found in this study applied to a population of homeless persons in London, a modest number of false-positive cases will be referred and over 60 cases of pulmonary tuberculosis identified. Some of the falsepositive cases may have old tuberculosis scars and will have the diagnosis excluded using bacterial culture, clinical and laboratory evaluation. Digital radiographs are easy to transmit electronically and use a lower dose of radiation.

Our findings suggest that highly trained readers may be able to improve the detection of tuberculosis in high-prevalence, hardto-reach, population groups. Digital chest radiographic screening of these groups may, therefore, be an important component of a holistic approach to the control of tuberculosis, which includes rapid detection and confirmation of infectious cases, and supportive measures to ensure treatment completion.

I. Abubakar*, A. Story*, M. Lipman", G. Bothamley , R. van Hest $^{+}$, N. Andrews*, J.M. Watson* and A. Hayward ${ }^{\S}$

Health Protection Agency Centre for Infections, Colindale, "Royal Free Hospital, "Homerton University Hospital NHS Foundation Trust, ${ }^{\S}$ Dept of Population Sciences and Primary Care, University College London, London, UK, and ${ }^{+}$Tuberculosis Control Section, Dept of Infectious Disease Control, Rotterdam Public Health Service, Rotterdam, Schiedamsedijk 95, 3011 EN, the Netherlands.

Correspondence: I. Abubakar, Tuberculosis Section, Respiratory and Systemic Infections Dept, Centre for Infections, Health Protection Agency, London, NW9 5EQ, UK. E-mail: Ibrahim.abubakar@hpa.org.uk

Statement of Interest: None declared. 
Acknowledgements: We would like to acknowledge the 13 medical consultants who assessed the films. We are grateful to L. Bradshaw (TB Section, Health Protection Agency Centre for Infections, London, UK) for proofreading the manuscript. No external funding was received for this study.

\section{REFERENCES}

1 Coker R, Bell A, Pitman R, et al. Tuberculosis screening in migrants in selected European countries shows wide disparities. Eur Respir J 2006; 27: 801-807.

2 Story A, Murad S, Verheyen M, et al. Tuberculosis in London - the importance of homelessness, problem drug use and prison. Thorax 2007; 62: 667-671.

3 World Health Organisation. Western Pacific Region. Assessing tuberculosis prevalence through population-based surveys. Geneva, World Health Organization, 2007.

4 Balabanova Y, Coker R, Fedorin I, et al. Variability in interpretation of chest radiographs among Russian clinicians and implications for screening programmes: observational study. BMJ 2005; 331: 379-382.

5 Shaw NJ, Hendry M, Eden OB. Inter-observer variation in interpretation of chest X-rays. Scott Med J 1990; 35: 140-141.

6 Nyboe J. Results of the international study on x-ray classification. Bull Int Union Tuberc 1968; 41: 115-124.

7 den Boon S, White NW, van Lill SW, et al. An evaluation of symptom and chest radiographic screening in tuberculosis prevalence surveys. Int J Tuberc Lung Dis 2006; 10: 876-882.

8 den Boon S, Bateman ED, Enarson DA, et al. Development and evaluation of a new chest radiograph reading and recording system for epidemiological surveys of tuberculosis and lung disease. Int J Tuberc Lung Dis 2005; 9: 1088-1096.

9 Graham S, Das GK, Hidvegi RJ, et al. Chest radiograph abnormalities associated with tuberculosis: reproducibility and yield of active cases. Int J Tuberc Lung Dis 2002; 6: 137-142.

10 Zellweger JP, Heinzer R, Touray $M$, et al. Intra-observer and overall agreement in the radiological assessment of tuberculosis. Int J Tuberc Lung Dis 2006; 10: 1123-1126.

11 de Vries G, van Hest RA, Richardus JH. Impact of mobile radiographic screening on tuberculosis among drug users and homeless persons. Am J Respir Crit Care Med 2007; 176: 201-207.

12 Crofts JP, Gelb D, Andrews N, et al. Investigating tuberculosis trends in England. Public Health 2008; 122: 1302-1310.

13 CDC. Instructions to panel physicians for completing chest $\mathrm{x}$-ray and classification worksheet (DS-3024). www.cdc.gov/ncidod/ dq/dsforms/3024.htm Date last updated: August 9, 2006. Date last accessed: June 4, 2008.

14 Nakamura K, Ohmi A, Kurihara T, et al. [Studies on the diagnostic value of $70 \mathrm{~mm}$ radiophotograms by mirror camera and the reading ability of physicians.] Kekkaku 1970; 45: 121-128.

15 Frieden T. Toman's Tuberculosis. Case Detection, Treatment and Monitoring. Geneva, World Health Organisation, 2004; pp. 92-95.

16 Dasgupta K, Menzies D. Cost-effectiveness of tuberculosis control strategies among immigrants and refugees. Eur Respir J 2005; 25 1107-1116.

17 Tulsky JP, Hahn JA, Long HL, et al. Can the poor adhere? Incentives for adherence to TB prevention in homeless adults. Int $J$ Tuberc Lung Dis 2004; 8: 83-91.

\section{Importance of identifying Mycobacterium bovis as a causative agent of human tuberculosis}

\section{To the Editors:}

The case study described hereafter emphasises the potentially vital importance of Mycobacterium bovis identification for appropriate tuberculosis patient management.

M. bovis, the classical causative agent of bovine tuberculosis, can be responsible for human TB, which makes this bacterium an important zoonotic species. In developed countries, the introduction of pasteurisation, preventing contamination from milk of infected cows, and eradication programmes for infected herds have considerably reduced the prevalence of human disease due to the bovine TB bacillus, but have not completely eradicated it [1-3]. Nevertheless, in many developed countries, the possibility of TB due to M. bovis infection, instead of Mycobacterium tuberculosis, is considered unlikely or even disregarded by microbiologists and clinicians.

Distinction of $M$. bovis from $M$. tuberculosis has significant relevance to patient management. In contrast to the other members of the $M$. tuberculosis complex, M. bovis is intrinsically resistant to pyrazinamide [4]. Beyond its use for specific $M$. bovis identification, this natural resistance is particularly important to consider. Pyrazinamide is usually given in the classical first-line TB treatment, as it is an effective sterilising drug that helps to shorten TB therapy due to its synergistic effect with rifampicin [5]. Thus, in case of M. bovis infection, pyrazinamide would be ineffective if implemented in a patient's anti-TB regimen. Unfortunately, results of in vitro susceptibility assays to pyrazinamide by conventional methods are not systematically correlated to in vivo activity. Susceptibility is, therefore, not often routinely assayed in laboratories. In addition, TB due to $M$. bovis is clinically, radiologically and microscopically indistinguishable from disease caused by $M$. tuberculosis. After culture on solid media, M. bovis displays specific characteristics like dysgonic colonies, and negative biochemical test results for nitrate reduction and niacin accumulation. However, clinical laboratories are increasingly using automated liquid culture systems followed by molecular tests for faster isolation and identification. Classical biochemical tests to identify $M$. bovis are not applicable using such liquid media, and most classical molecular identification methods based on targets like IS6110, 16S rDNA, 23S rDNA or ITS, do not distinguish between $M$. bovis and the other members of the $M$. tuberculosis complex. Other genetic markers [6] and the single commercial test (GenoType Mycobacterium, Hain, Nehren, Germany) allowing distinction between $M$. tuberculosis complex members are not widely used. 